\title{
SYNTHESIS AND REACTIONS OF SOME SPIROFUROCHROMANONE DERIVATIVES WITH POTENTIAL BIOLOGICAL ACTIVITY
}

\author{
S.I. E1-Desoky \\ University of Mansoura, Faculty of Science, Chemistry Department
}

\begin{abstract}
Condensation of differnet spiro furochromanone derivatives la-c with malononitrile afforded $N\left(l^{\prime}\right.$-cyanoethyl-2',2'-dicyanovinyl) chromen-5-amine $2 a, b$ and the ylidene malonontrile $3 a-c$ derivatives according to the reaction conditions. Reaction of the latter products with hydrazine hydrate phenyl hydrazine and thiourea gave the products 4-8. Reaction of the ylidene 3a-c with sulphur and triethylamine to give the product 9 a-c. The latter were reacted with different aromatic aldehyde, acetic anhydride, diethylmalonate and dimethyl acetylenedicarboxylate to give the product 10-14.
\end{abstract}

\section{Introduction}

The naturally occurring furobenzopyran and benzofuran rings as the basic skeleton of nuemrous compounds possessing cardiovascular and antiarrhythmic activity $(1-3)$. Benzopyranone used as sapasmolytic, cytotoxic and hepatotoxic and antidiabetic $(4,5)$. Also, furochromone derivatives are used as antispamodics for the relief of spasms of the ureter, bile duct, gall bladder and bronchial asthma (6).

\section{Chemistry and Discussion}

Condensation of spirofurochromanones derivatives $\mathbf{l a}$ or $\underline{1} \mathbf{b}$. (7) with malononitrile afforded two different products according to the reaction conditions, at using ethanolic sodium ethoxide solution gave spirocyclohexyl furochromen-5-amine derivatives $\mathbf{2 a} \cdot \mathbf{b}$ via dimerization of malonitrile before its condensation in one pot reaction, whereas in acetic acid/ammonium acetate mixture afforded the ylidene malononitrile derivatives $3 \mathbf{a}-\mathbf{c}$. ${ }^{1} \mathrm{H}$ NMR spectrum of compound $2 \mathbf{b}$ showed clearly a singlets signals at $\delta=3.54,5.16$ and 11.98 for $\mathrm{CH}_{2}, \mathrm{CH}$ and $\mathrm{NH}$ protons respectively.

The ylidene malononitrile $\mathbf{3 a}, \mathbf{b}$ were reacted with hydrazine hydrate to afford the diylidene hydrazine 4 which probably from Michael addition to $\alpha, \beta$ unsaturated nitrile followed by extrusion malononitrile molecule. The same compound $\underline{4}$ cannot be obtained directly from reaction of $1 \underline{b}$ with hydrazine hydrate. In another attempt, at room temperature the diamino pyrazole derivative $\mathbf{5}$ was separated. ${ }^{1} \mathrm{H}$ NMR spectra of the compounds 4 and 5 showed the methylene protons at $\delta=3.1$ and $2.6 \mathrm{ppm}$ also a two amino groups was appeared as broad peaks at $\delta=5.4$ and $6.4 \mathrm{ppm}$.

The ylidene malononitrile $3 \mathrm{~b}$ reacted with phenyl hydrazine to give the corresponding furochroman-5-yl pyrazole derivatives 6 . Whereas refluxing a mixture of $\mathbf{3 a}$ and thiourea in sodium ethoxide solution, the diaminopyrimidinthione derivative $\mathbf{7}$ not obtianed, instead compound 8 , was separated which clearly established from a characteristic cyano absorption band at $v=2190$. 


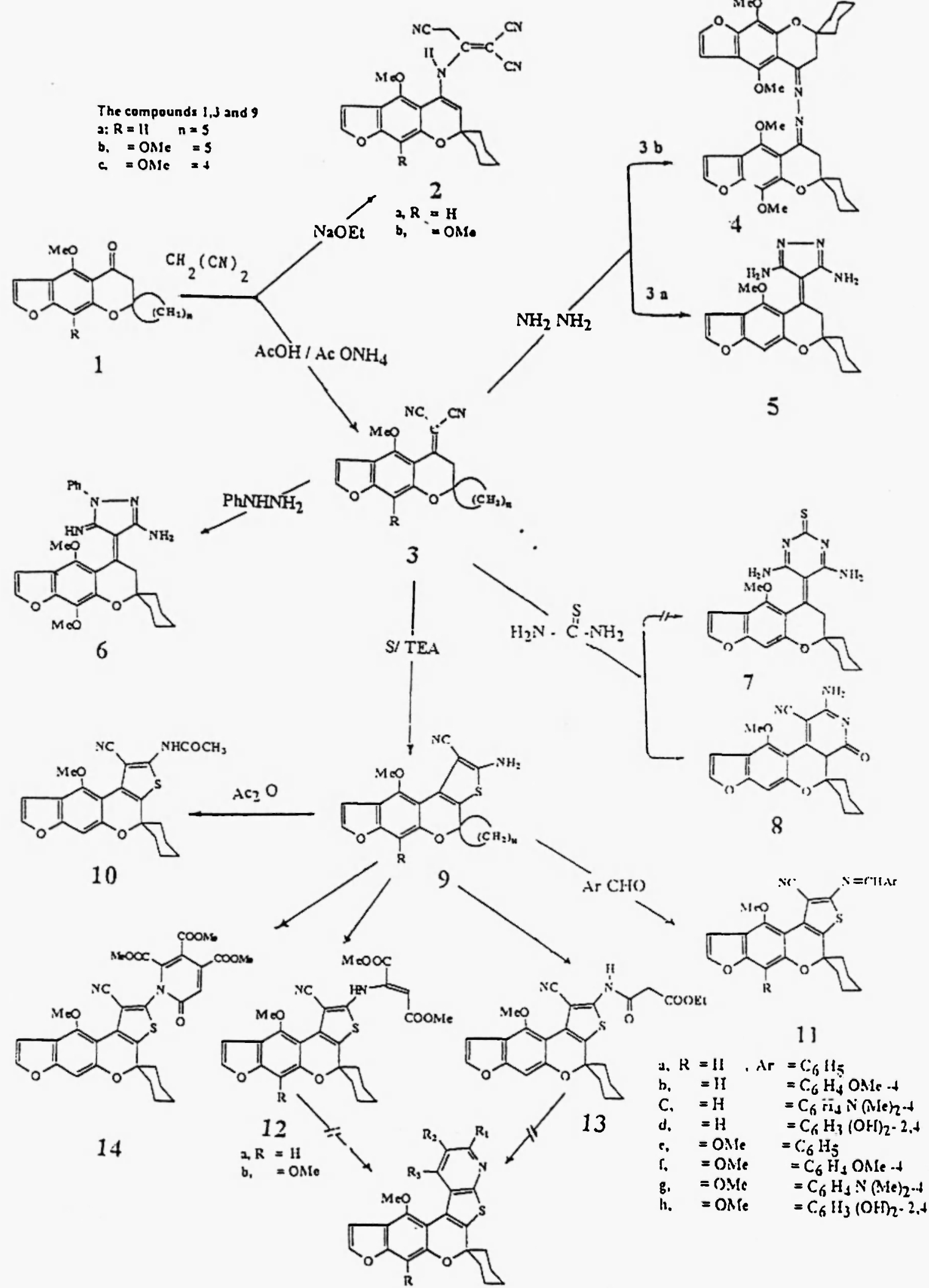


Also, at reaction of $3 \mathrm{a}-\mathrm{c}$ with sulphur and triethylamine gave a quantitatively yield from the corresponding 6-amino-5-cyano-4-methoxy furo $[3,2 \mathrm{~g}]$ thieno[2,3-c] chromen derivatives 9a-c. $\mathrm{N}$-acylation of 9a yielded 6-acetamido-5-cyano-4-methoxy-7-pentamethylene furo[3,2-g]thieno[2,3c]chromen 10, which showed in $\mathrm{IR}$ spectrum disappearance of $\mathrm{NH}_{2}$ signals.

The above products $\underline{9} \underline{a}$ or $\underline{9} \mathbf{b}$ were condensed with benzaldehyde, $\mathrm{p}$-anisaldehyde, P-N.Ndimethylbenzaldehyde and 2,4-dihydroxy benzaldehyde to give the corresponding products $11 \mathbf{a}-\underline{\mathbf{h}}$. Also, reaction of $9 \mathbf{a}$ or $\underline{9 b}$ with dimethyl acetylene dicarboxylate and diethyl malonate in different basic medium, compound 15 not obtained instead, the compounds $12-14$ were separated.

\section{Experimental}

All melting points are uncorrected. The IR spectra were recorded on a Mattson 500 FTTR spectrometer using in all sample $\mathrm{KBr}$ disk and elemental analyses were carried out in the Microanalytical Unit (Faculty of Science, Mansoura University). The ${ }^{{ }^{l}} \mathrm{H}$ NMR were recorded on Varian - Gemini $200 \mathrm{MHz}$ using TMS as internal standard and $\mathrm{CDCl}_{3}$ as solvent.

\section{Reaction of malononitrile with compound 1a-c.}

A: Preparation of N-(1'-cyanomethyl-2',2'-dicyanovinyl) 4-methory (and 4,9-dimethoxy)-7pentamethylene furo[3,2-g]8H-Chromen-5-amine $2 \mathrm{a}$ and $2 \mathrm{~b}$.

A mixture of compound $1 \mathbf{a}$ or $\mathbf{1 b}$. (7) $(10 \mathrm{mmol})$ and malononitrile $(20 \mathrm{mmol})$ in absolute ethanol $(30 \mathrm{ml})$ were refluxed with stirring. Sodium ethoxide solution $(0.4 \mathrm{~g}$ in $10 \mathrm{ml}$ absolute ethanol) was dropwisely added through $15 \mathrm{~min}$ then heating was continued for $5 \mathrm{hrs}$. The reaction mixture was poured into crushed ice after cooling and neutralized with diluted hydrochloric acid. The precipitate formed was filtered off, washed with water and crystallized from ethanol.

IR (compound 2a) $v=3131(\mathrm{NH}), 2994,2866(\mathrm{CH}), 2219(\mathrm{C} \equiv \mathrm{N})$ and $1624 \mathrm{~cm}^{-1}$.

IR (Compound $2 \underline{b}$ ) $v=3100(\mathrm{NH}), 2932,2859(\mathrm{CH}), 2220(\mathrm{C} \equiv \mathrm{N}) 1612(\mathrm{C}=\mathrm{N}), 1584(\mathrm{Ar}$ ).

$1_{\mathbf{H}} \mathrm{NMR}$ (Compound 2b) $\delta=1.2-2.1\left(\mathrm{~m}, 10 \mathrm{H}\right.$, five $\left.\mathrm{CH}_{2}\right), 3.84\left(\mathrm{~s}, 2 \mathrm{H}, \mathrm{CH}_{2} \mathrm{CN}\right), 4.06(\mathrm{~s}, 3 \mathrm{H}$, $\left.\mathrm{OCH}_{3}\right), 4.18\left(\mathrm{~s}, 3 \mathrm{H}, \mathrm{OCH}_{3}\right) 5.16(\mathrm{~s}, 1 \mathrm{H}, \mathrm{H}-6), 6.92(\mathrm{~d}, 1 \mathrm{H}, \mathrm{H}-3), 7.53(\mathrm{~d}, 1 \mathrm{H}, \mathrm{H}-2), 11.98(\mathrm{~s}, 1 \mathrm{H}$, $\mathrm{NH})$.

\section{B: Preparation of the ylidene malononitrile 3a-c "general procedure"}

A mixture of compound $1 \mathrm{a}-\mathrm{c}(10 \mathrm{mmol})$, malononitirle $(10 \mathrm{mmol})$, ammonium acetate $(3 \mathrm{~g})$ and acetic acid $(3 \mathrm{ml})$ were refluxed in chloroform $(200 \mathrm{ml})$ using dean stark apparatus for $8 \mathrm{hrs}$. The solvents were evaporated under vacuum then water $(100 \mathrm{ml})$ was added. The precipitate formed was filtered off, washed with water and crystallized from ethanol

IR (Compound 3a) $v=2931,2853(\mathrm{CH}), 2209(\mathrm{C} \equiv \mathrm{N})$ and $1613 \mathrm{~cm}^{-1}$.

IR (Compound $3 \mathrm{~b}) v=2936,2852(\mathrm{CH}), 2224(\mathrm{C} \equiv \mathrm{N})$ and $1622 \mathrm{~cm}^{-1}(\mathrm{C}=\mathrm{C})$.

IR (Compound 3c) v=3131, 2950(CH), 2223(C $\equiv N)$ and $1612 \mathrm{cn}^{-1}(\mathrm{C}=\mathrm{C})$. 


\section{Reaction of $\underline{3 a, b}$ with hydrazine hydrate}

A: Preparation of di(4,9-dimethoxy-7-pentamethylene furo(3,2-g) chroman-5-ylidene) hydrazine (4)

A mixture of compound $\underline{3} \underline{\mathbf{b}}(10 \mathrm{mmol})$ and hydrazine monohydrate $(1 \mathrm{ml}, 98 \%)$ was boiled in absolute ethanol for $6 \mathrm{hrs}$. The reaction mixture was evaporated and the residue was crystallized from ethanol.

IR $v=2930,2860(\mathrm{CH}), 1616(\mathrm{C}=\mathrm{N})$ and $1591 \mathrm{~cm}^{-1}(\mathrm{Ar})$.

$1_{\mathbf{H}}$ NMR $\delta=1.5-2.4\left(\mathrm{~m}, 20 \mathrm{H}\right.$, Ten $\mathrm{CH}_{2}$ groups), 3.11(s, 4H, Two $\left.\mathrm{CH}_{2}\right), 4.02(2 \mathrm{~s}, 12 \mathrm{H}$ four $\left.\mathrm{OCH}_{3}\right) 6.88(\mathrm{~d}, 2 \mathrm{H}$, two $\mathrm{H}-3)$ and $7.5 \mathrm{ppm}(\mathrm{d}, 2 \mathrm{H}, \mathrm{H}-2)$.

B: Preparation of 3,5-diamino-4(4'-methoxy-7'-pentamethylene-furo[3',2'-g].Chroman-5ylidene)-4H-pyrazole 5

Hydrazine hydrate $(1 \mathrm{ml}, 98 \%)$ was added dropwise to a stirred solution of $2 \mathrm{a}$ ( $(3.34 \mathrm{~g} 10$ $\mathrm{mol}$ ) in absolute ethanol through $10 \mathrm{~min}$. The reaction mixture was stirred at room temperature with controlling the time of reaction using TLC for about $30 \mathrm{~min}$. Then poured into crushed ice $(30 \mathrm{~g})$, filtered off and crystallized from ethanol.

IR $v=3363,3287,3213,3132\left(2 \mathrm{NH}_{2}\right.$ groups), 2986, 1855(CH), $1620(\mathrm{C}=\mathrm{N})$ and $1579 \mathrm{~cm}^{-1}$ (Ar.). $1_{\mathbf{H}} \mathrm{NMR} \delta=1.2-2.0\left(\mathrm{~m}, 10 \mathrm{H}\right.$, five $\left.\mathrm{CH}_{2}\right), 2.57(\mathrm{~s}, 2 \mathrm{H}, \mathrm{H}-6), 3.99(\mathrm{~s}, 3 \mathrm{H}, \mathrm{OMe}), 5.15,6.20(2 \mathrm{~s}$, broad, $4 \mathrm{H}$, two $\mathrm{NH}_{2}$ protons), 6.84(d, $\left.1 \mathrm{H}, \mathrm{H}-3\right)$ and $7.44 \mathrm{ppm}(\mathrm{d}, 1 \mathrm{H}, \mathrm{H}-2)$.

Preparation of 1-phenyl-3-amino-4-(4, ,9-dimethoxy-7-pentamethylene furo[3",2"-g]chroman 5-ylidene)4H-pyrazole 6

A mixture of compound $3 \mathrm{~b}$. $(5 \mathrm{mmol})$ and phenyl hydrazine $(6 \mathrm{mmol})$ in absolute ethanol was boiled under reflux for 5 hrs. The precipitate was formed after cooling, filtered off, washed with water, dried and crystallized from ethanol.

IR $v=3360,3218\left(\mathrm{NH}_{2}\right), 3210(\mathrm{NH}), 1616(\mathrm{C}=\mathrm{N})$ and $1591 \mathrm{~cm}^{-1}(\mathrm{Ar})$.

$1_{\mathbf{H}} \mathrm{NMR} \delta=1.41-2.01\left(\mathrm{~m}, 10 \mathrm{H}\right.$, five $\left.\mathrm{CH}_{2}\right), 2.66(\mathrm{~s}, 2 \mathrm{H}, \mathrm{H}-6), 4.03,4.10(2 \mathrm{~s}, 6 \mathrm{H}$, two $\mathrm{OMe}), 6.88(\mathrm{~s}$, $2 \mathrm{H}, \mathrm{NH}_{2}$ ), 7.22(d, lH, H-3), 7.25-7.33(m, 5H, Ar-H), 7.48(d, 1H, H-2).

\section{Reaction of compound 3a with thiourea "preparation of compound 8 "}

Sodium ethoxide solution $(0.3 \mathrm{~g} / 10 \mathrm{ml}$ absolute ethanol) was added with stirring to a mixture from compound $3 \mathrm{a} .(1.67 \mathrm{~g}, 5 \mathrm{mmol})$ and thiourea $(0.51 \mathrm{~g}, 8 \mathrm{mmol})$ in ethanol $(30 \mathrm{ml})$. The mixture was boiled for $4 \mathrm{hrs}$, then poured into cooled water $(100 \mathrm{ml})$ and neutralized with diluted hydrochloric acid. The precipitate was collected, filtered off and crystallized from ethanol.

IR $v=3418,3260,3137\left(\mathrm{OH}, \mathrm{NH}_{2}\right), 3380-3000$ (br., enolic $\left.\mathrm{OH}\right), 2924,2859$ (CH), 2190(sharp 
strong $C \equiv N), 1660,(C=0)$ and, $1613 \mathrm{~cm}^{-1}(\mathrm{Ar})$.

\section{Synthesis of the compound 9a-c}

A solution of the ylidene malononitrile $\underline{3 a-c}(10 \mathrm{mmol})$, sulfur metal $(0.4 \mathrm{~g}, 12 \mathrm{mmol})$ in absolute ethanol $(50 \mathrm{ml})$ was boiled with stirring. Triethylamine $(2 \mathrm{ml})$ was dropwisely added then heat the mixture for $6 \mathrm{hrs}$. The reaction mixture was concentrated in vacuo and the residue formed was crystallized from ethanol.

IR (Compound 9a) v=3450, 3337, 3201( $\left.\mathrm{NH}_{2}\right), 2933,2859(\mathrm{CH}), 2197(\mathrm{C} \equiv \mathrm{N})$ and $1619, \mathrm{~cm}^{-1}$ (Ar.) ${ }^{1} \mathrm{H}$ NMR $\delta=1.26-2.20\left(\mathrm{~m}, 10 \mathrm{H}\right.$, five $\left.\mathrm{CH}_{2}\right), 4.26\left(\mathrm{~s}, 3 \mathrm{H}, \mathrm{OCH}_{3}\right), 4.85\left(\mathrm{~s}\right.$, br. $\left.2 \mathrm{H}, \mathrm{NH}_{2}\right), 6.88(\mathrm{~s}, 1 \mathrm{H}$, $\mathrm{H}-10)$ 6.98(d, 1H, H-3) and $7.47 \mathrm{ppm}(\mathrm{d}, 1 \mathrm{H}, \mathrm{H}-2)$.

MS: $\mathrm{M}^{+}(\mathrm{m} / \mathrm{z}=366)$.

IR (Compound 9b) $v=3410,3320,3210\left(\mathrm{NH}_{2}\right), 2936,2847(\mathrm{CH})$ and $2201 \mathrm{~cm}^{-1}(\mathrm{C} \equiv \mathrm{N})$

IR (Compound 9c) $v=3410,3240\left(\mathrm{NH}_{2}\right), 2205(\mathrm{C} \equiv \mathrm{N})$ and 1612 (Ar.).

Synthesis of 6-acetamido-5-cyano-4-methoxy-8-pentamethylene furo $[3,2-\mathrm{g}]$ thiano|2,3-c]8H Chromen (10)

A mixture of compound $9 \mathbf{a}(1 \mathrm{~g}, 2.7 \mathrm{mmol})$ and acetic anhydride $(0.4 \mathrm{ml}, 4 \mathrm{mmol})$ was boiled in acetic acid $(10 \mathrm{ml})$ for $8 \mathrm{hrs}$. The reaction mixture was poured into crushed ice and the precipitate formed was filtered off, washed with water and crystallized from ethanol. IR $v=3273(\mathrm{NH}), 2930,(\mathrm{CH}), 2211\left(\mathrm{C} \equiv \mathrm{N}\right.$ ) 1692(amide I), 1623(amide II) and $1585 \mathrm{~cm}^{-1}$ (Ar.).

Condensation of compound $\underline{8}$ with aromatic aldehydes, preparation of the compounds $11 \mathrm{a}-\mathrm{h}$ "general procedure"

A mixture of compound 9a or $9 \mathrm{~b}$. $(3 \mathrm{mmol})$, the corresponding aldehyde $(3 \mathrm{mmol})$ and triethyl amine $(0.5 \mathrm{ml})$ in ethanol $(30 \mathrm{ml})$ was refluxed for a time $3-5 \mathrm{hrs}$. The precipitate formed after cooling was filtered off, dried and crystallized from ethanol.

IR (Compound 11a) $v=2930,2860(\mathrm{CH}), 2220(\mathrm{C} \equiv \mathrm{N})$ and $1610 \mathrm{~cm}^{-1}(\mathrm{C}=\mathrm{N})$.

IR (Compound 11 b) $v=2935,2854(\mathrm{CH}), 2222(\mathrm{C} \equiv \mathrm{N}), 1600(\mathrm{C}=\mathrm{N}), 1566(\mathrm{Ar})$.

IR (Compound 11c) $v=2927,2856(\mathrm{CH}) 2210\left(\mathrm{C} \equiv \mathrm{N}\right.$ and $1618 \mathrm{~cm}^{-1}$

IR (Compound 11d) $v=3500-3200(\mathrm{br}, \mathrm{OH}$ bonded), 3170, $3130(\mathrm{OH}$ free), 2933, $2847(\mathrm{CH})$, $2220(\mathrm{C} \equiv \mathrm{N}), 1626(\mathrm{C}=\mathrm{N})$ and $1603 \mathrm{~cm}^{-1}(\mathrm{Ar})$.

IR (Compound 11e) $v=2935,2849(\mathrm{CH}), 2210(\mathrm{C} \equiv \mathrm{N})$ and $1605 \mathrm{~cm}^{-1}(\mathrm{C}=\mathrm{N})$.

IR (Compound 110) $v=2935,2843(\mathrm{CH}), 2216(\mathrm{C} \equiv \mathrm{N}), 1591(\mathrm{C}=\mathrm{N})$ and $1566 \mathrm{~cm}^{-1}(\mathrm{Ar}$.)

IR (Compound 11g) $v=2932,2840(\mathrm{CH}), 1613(\mathrm{C}=\mathrm{N}), 1578$ (Ar.) 1533(Sh. strong N(Me) $)_{2}$ )

${ }^{1} \mathrm{H}$ NMR (Compound $\underline{11 \mathrm{~g})} \delta=1.25-2.1\left(\mathrm{~m}, 10 \mathrm{H}\right.$, five $\left.\mathrm{CH}_{2}\right), 3.09\left(\mathrm{~s}, 6 \mathrm{H}, \mathrm{N}\left(\mathrm{CH}_{3}\right)_{2}\right), 4.1(\mathrm{~s}, 3 \mathrm{H}$, $\left.\mathrm{OCH}_{3}\right), 4.22\left(\mathrm{~s}, 3 \mathrm{H}, \mathrm{OCH}_{3}\right), 6.71(\mathrm{~d}, 2 \mathrm{H}, \mathrm{J}=45 \mathrm{~Hz}$ Two H-3'), 7.02(d, $1 \mathrm{H}, \mathrm{J}=11 \mathrm{~Hz} \mathrm{H}-3), 7.52(\mathrm{~d}$, 
$1 \mathrm{H}, \mathrm{J}=11 \mathrm{~Hz} \mathrm{H}-2), 7.86\left(\mathrm{~d}, 2 \mathrm{H} \mathrm{J}=45 \mathrm{~Hz}, \mathrm{Two} \mathrm{H}-2^{\prime}\right)$ and $8.37 \mathrm{ppm}(\mathrm{s}, 1 \mathrm{H}, \mathrm{N}=\mathrm{CH})$.

IR (Compound 11h) $v=3600-3100($ br., OH), 2935, 2858(CH), $2220(\mathrm{C}=\mathrm{N}), 1619(\mathrm{C}=\mathrm{N}), 1600$, 1574 (Ar.).

\section{Reaction of compound $\underline{7}$ with dimethyl acetylenedicarboxylate or diethylmalonate}

\section{Preparation of the compounds 12-13 "General procedure"}

Sodium ethoxide solution $(0.5 \mathrm{~g}$ in $10 \mathrm{ml}$ absolute ethanol) was dropwisely added to a mixture from compound $9 \mathbf{a}$ or $9 \mathbf{b}(10 \mathrm{mmol})$ and dimethyl acetylenedicarboxylate (or diethylmalonate) $(12 \mathrm{mmol})$ in absolute ethanol $(50 \mathrm{ml})$. The mixture was refluxed with stirring for 8 hrs, cooled, poured into crushed ice and neutralized with diluted hydrochloric acid. The precipitate formed was filtered off and crystallized from ethanol.

IR (Compound 12a) $v=2937,2857(\mathrm{CH}), 2220(\mathrm{C} \equiv \mathrm{N}), 1736\left(\mathrm{COOCH}_{3}\right)$ and $1621 \mathrm{~cm}^{-1}(\mathrm{C}=\mathrm{C})$.

IR (Compound 12b) $v=2220(\mathrm{C} \equiv \mathrm{N}), 173 \mathrm{I}\left(\mathrm{COOCH}_{3}\right), 1610(\mathrm{C}=\mathrm{C}) 1560(\mathrm{Ar})$

IR (Compound 13) $v=3236(\mathrm{NH}), 3115,2930,2856(\mathrm{CH}), 2221(\mathrm{C} \equiv \mathrm{N}), 1730(\mathrm{COOEt}), 1668$ (amide I), 1624 (amide II) and $1583 \mathrm{~cm}^{-1}$ (Ar)

${ }^{1} \mathrm{H}$ NMR (Compond 13) $\delta=1.35\left(\mathrm{t}, 3 \mathrm{H}, \mathrm{CH}_{3} \mathrm{CH}_{2}\right), 141-2.17\left(\mathrm{~m}, 10 \mathrm{H}\right.$, five $\left.\mathrm{CH}_{2}\right), 3.62(\mathrm{~s}, 3 \mathrm{H}$. $\left.\mathrm{OCH}_{3}\right), 4.28\left(\mathrm{~s}, 2 \mathrm{H}, \mathrm{COCH}_{2} \mathrm{CO}\right), 4.33\left(\mathrm{q}, 2 \mathrm{H}, \mathrm{CH}_{3} \mathrm{CH}_{2}\right), 6.90(\mathrm{~s}, 1 \mathrm{H}, \mathrm{H}-10), 7.01(\mathrm{~d}, 1 \mathrm{H}, \mathrm{H}-3)$. $7.47(\mathrm{~d}, 1 \mathrm{H}, \mathrm{H}-2), 10.95(\mathrm{~s}, 1 \mathrm{H}$, amide $\mathrm{NH})$

\section{Preparation of compound 14}

A mixture from compound $9(1.8 \mathrm{~g}, 5 \mathrm{mmol})$, dimethyl acetylene dicarboxylate $(2.1 \mathrm{~g}, 15$ $\mathrm{mmol})$ and unhydrous potassium carbonate $(\mathrm{g})$ in dimethyl sulphoxide $(20 \mathrm{ml})$ was refluxed for : hrs. The reaction mixture was worked up as previously discussed in preparation of the compounds $12-13$ to give compound 14 as pale yellow crystals

IR $v=2941,2855(\mathrm{CH}), 2220(\mathrm{C} \equiv \mathrm{N}), 1740(\mathrm{COOMe})$ and $1700 \mathrm{~cm}^{-1}(\mathrm{C}=\mathrm{O})$.

${ }^{1} \mathrm{H}$ NMR $\delta=1.25-2.35\left(\mathrm{~m}, 10 \mathrm{H}\right.$, five $\left.\mathrm{CH}_{2}\right), 3.70,3.83,3.93$ (3 singlets, $9 \mathrm{H}$, Three $\mathrm{COOCH}_{3}$ ). $4.27\left(\mathrm{~s}, 3 \mathrm{H}, \mathrm{OCH}_{3}\right) \quad 6.93(\mathrm{~s}, \mathrm{IH}, \mathrm{CH}$ of pyridone), $7.01(\mathrm{~d}, 1 \mathrm{H}, \mathrm{J}=12 \mathrm{~Hz} \mathrm{H}-3)$ and $7.50 \mathrm{ppm}$ (d, $1 \mathrm{H} \mathrm{J}=12 \mathrm{~Hz}, \mathrm{H}-2$ )

\section{Referneces}

1) F. Binon, Chim Ther., 7, 156 (1972).

2) Guy Bourgery, Philippe Dostert, Alain lacour, Michel langlois, Bermard Pourrias and Jacky Tisne - Versailles J. Med. Chem. 24, 159 (1981).

3) B. Pourrias and F. Friederich, Eur. J. Pharmacol 49, 203 (1978).

4) Virinder s. Parmar; Sandhya Gupta; Rakesh, K. Sharma and Varun, K. Sharma. J Org Chem 55, 1193 (1990). 
5) Ronald B. Gammill and Bruce R. Hyde J. Org. Chem. 48, 3963 (1983).

6) A Mustafa "Furopyranes and Furopyrones in the Chemistry of Heterocyclic Compounds", Ed. A Weisberger Interscience Publisher. J. Wiley and Sons, London (1967) and references therein.

7) El-Desoky, S.I; M.A. Hammad; Grant, N., El-Telbany, E.M. and Abdel-Rahman, A.H. under publication

\section{Received October 28, 1996}

Table I Characterization data of the new prepancel compounds

\begin{tabular}{|c|c|c|c|c|c|c|c|c|c|}
\hline \multirow{3}{*}{$\begin{array}{c}\text { Comp } \\
\text { No }\end{array}$} & \multirow[t]{3}{*}{ M.P. ${ }^{\circ} \mathrm{C}$} & \multirow{3}{*}{$\begin{array}{c}\text { Yield } \\
\%\end{array}$} & \multirow{3}{*}{ Mol formula mol weight) } & \multicolumn{6}{|c|}{ Analysis } \\
\hline & & & & \multicolumn{3}{|c|}{ Calcd } & \multicolumn{3}{|c|}{ Found } \\
\hline & & & & C & $\mathrm{HH}$ & $\mathrm{N}$ & C & $\mathrm{H}$ & $\mathrm{N}$ \\
\hline $2 a$ & $126-28$ & 81 & $\mathrm{C}_{23} \mathrm{H}_{20} \mathrm{~N}_{4} \mathrm{O}_{3}(400.44)$ & 69.00 & 5.03 & 14.00 & 69.16 & 5.22 & 13.78 \\
\hline $2 b$ & $105-8$ & 80 & $\mathrm{C}_{24} \mathrm{H}_{22} \mathrm{~N}_{4} \mathrm{O}_{4}(43046)$ & 6697 & 5.15 & 13.02 & 6685 & 5.34 & 13.28 \\
\hline 3a & $125-27$ & 93 & $\mathrm{C}_{20} \mathrm{ll}_{18} \mathrm{~N}_{2} \mathrm{O}_{3}(334.37)$ & 7184 & 5.34 & 8.38 & 71.69 & 5.61 & 8.18 \\
\hline $3 b$ & $134-36$ & 95 & $\mathrm{C}_{2} \mathrm{I}_{20} \mathrm{~N}_{2} \mathrm{O}_{4}(364.40)$ & 69.22 & 5.53 & 768 & 69.41 & 5.47 & 7.82 \\
\hline $3 c$ & $127-29$ & 85 & $\mathrm{C}_{20} \mathrm{H}_{18} \mathrm{~N}_{2} \mathrm{O}_{4}(350.37)$ & 68.56 & 5.18 & 8.00 & 68.70 & 5.35 & 7.99 \\
\hline 4 & $197-99$ & 80 & $\mathrm{C}_{36} \mathrm{H}_{40} \mathrm{~N}_{2} \mathrm{O}_{8}(628.72)$ & 6877 & 6.41 & 446 & 68.91 & 6.61 & 4.37 \\
\hline 5 & $214-18$ & 82 & $\mathrm{C}_{20} \mathrm{H}_{22} \mathrm{~N}_{4} \mathrm{O}_{3}(366.42)$ & 65.56 & 605 & 1529 & 65.44 & 6.23 & 15.08 \\
\hline 6 & $178 \cdot 80$ & 90 & $\mathrm{C}_{27} \mathrm{H}_{28} \mathrm{~N}_{4} \mathrm{O}_{4}(472.54)$ & 68.53 & 597 & 11.85 & 68.37 & 5.79 & 11.94 \\
\hline 8 & $215-17$ & 76 & $\mathrm{C}_{2}{ }_{1} \mathrm{H}_{19} \mathrm{~N}_{3} \mathrm{O}_{4}(377.40)$ & 6683 & 5.07 & 11.13 & 6668 & 5.24 & 11.08 \\
\hline $9 a$ & $212-14$ & 90 & $\mathrm{C}_{20} \mathrm{H}_{18} \mathrm{~N}_{2} \mathrm{O}_{3} \mathrm{~S}(366.43)$ & 65.56 & 4.95 & 7.64 & 65.80 & 4.81 & 7.79 \\
\hline $9 b$ & $160-63$ & 88 & $\mathrm{C}_{21} \mathrm{H}_{20} \mathrm{~N}_{2} \mathrm{O}_{4} \mathrm{~S}(396.46)$ & 63.61 & 5.08 & 7.06 & 63.42 & 5.26 & 7.22 \\
\hline $9 c$ & $>300$ & 78 & $\mathrm{C}_{20} \mathrm{H}_{18} \mathrm{~N}_{2} \mathrm{O}_{4} \mathrm{~S}(382.43)$ & 62.81 & 4.74 & 7.33 & 62.72 & 489 & 7.41 \\
\hline 10 & $230-32$ & 76 & $\mathrm{C}_{23} \mathrm{H}_{22} \mathrm{~N}_{2} \mathrm{O}_{5} \mathrm{~S}(438.50)$ & 63.00 & 5.06 & 6.39 & 63.17 & 5.21 & 6.14 \\
\hline $11 \mathrm{a}$ & $205-8$ & 85 & $\mathrm{C}_{27} \mathrm{H}_{22} \mathrm{~N}_{2} \mathrm{O}_{3} \mathrm{~S}(454.54)$ & 7135 & 488 & 6.16 & 71.58 & 4.97 & 6.01 \\
\hline $11 \mathrm{~b}$ & $234-37$ & 90 & $\mathrm{C}_{28} \mathrm{H}_{24} \mathrm{~N}_{2} \mathrm{O}_{4} \mathrm{~S}(484.57)$ & 69.40 & 499 & 5.78 & 69.28 & 4.79 & 5.91 \\
\hline IIc & $268-70$ & 90 & $\mathrm{C}_{29} \mathrm{H}_{27} \mathrm{~N}_{3} \mathrm{O}_{3} \mathrm{~S}(497.61)$ & 70.00 & 5.47 & 8.44 & 70.17 & 5.32 & 8.32 \\
\hline $11 d$ & 268.70 & 93 & $\mathrm{C}_{27} \mathrm{H}_{22} \mathrm{~N}_{2} \mathrm{O}_{5} \mathrm{~S}(486.54)$ & 66.65 & 4.56 & 5.76 & 66.80 & 4.77 & 5.91 \\
\hline $11 \mathrm{e}$ & $228-31$ & 90 & $\mathrm{C}_{28} \mathrm{H}_{24} \mathrm{~N}_{2} \mathrm{O}_{4} \mathrm{~S}(484.57)$ & 6940 & 4.99 & 5.78 & 69.28 & 469 & 5.53 \\
\hline $11 f$ & 240.42 & 95 & $\mathrm{C}_{29} \mathrm{H}_{26} \mathrm{~N}_{2} \mathrm{O}_{5} \mathrm{~S}(514.59)$ & 67.69 & 5.09 & 5.44 & 76.69 & 5.31 & 5.28 \\
\hline $11 \mathrm{~g}$ & $248-50$ & 88 & $\mathrm{C}_{30} \mathrm{H}_{29} \mathrm{~N}_{3} \mathrm{O}_{4} \mathrm{~S}(527.64)$ & 68.29 & 5.54 & 7.96 & 68.08 & 5.29 & 7.77 \\
\hline $11 \mathrm{~h}$ & $>300$ & 85 & $\mathrm{C}_{28} \mathrm{H}_{24} \mathrm{~N}_{2} \mathrm{O}_{6} \mathrm{~S}(516.57)$ & 65.10 & 4.68. & 5.42 & 65.31 & 4.75 & 5.29 \\
\hline $12 \mathrm{a}$ & $190-92$ & 85 & $\mathrm{C}_{26} \mathrm{H}_{24} \mathrm{~N}_{2} \mathrm{O}_{7} \mathrm{~S}(508.54)$ & 61.41 & 4.76 & 5.51 & 61.66 & 458 & 5.80 \\
\hline $12 b$ & $230-33$ & 78 & $\mathrm{C}_{27} \mathrm{H}_{26} \mathrm{~N}_{2} \mathrm{O}_{8} \mathrm{~S}(538.57)$ & 60.21 & 4.87 & 5.20 & 60.19 & 4.72 & 5.43 \\
\hline $13 \mathbf{a}$ & $154-56$ & 83 & $\mathrm{C}_{25} \mathrm{H}_{24} \mathrm{~N}_{2} \mathrm{O}_{6} \mathrm{~S}(480.53)$ & 62.49 & 5.03 & 5.83 & 62.34 & 5.28 & 5.71 \\
\hline 15 & $182-84$ & 75 & $\mathrm{C}_{31} \mathrm{H}_{26} \mathrm{~N}_{2} \mathrm{O}_{10} \mathrm{~S}(618.61)$ & 60.19 & 4.24 & 4.53 & 60.03 & 4.43 & 4.39 \\
\hline
\end{tabular}


